Matteo Bassetti

Cristobal Leon Jean Francois Timsit

\section{Are prophylactic antifungals in highly colonized patients safe and effective?}

Received: 12 May 2015

Accepted: 15 May 2015

Published online: 3 June 2015

(C) Springer-Verlag Berlin Heidelberg and ESICM 2015

\section{Bassetti ( $)$}

Infectious Diseases Division, Santa Maria Misericordia University Hospital, Piazzale Santa Maria Della Misericordia 15,

33100 Udine, Italy

e-mail: mattba@tin.it

Tel.: +390432559353

C. Leon

CU-Valme University Hospital, University of Seville,

Avda Bellavista s/n, 41014 Seville, Spain

\section{J. F. Timsit}

Université Paris Diderot/Hopital Bichat, Réanimation

Medicale Et Des Maladies Infectieuses, Paris 75018, France

\section{J. F. Timsit}

UMR 1137, IAME Team 5, DeSCID: Decision Sciences in Infectious Diseases, Control and Care Inserm/Université Paris Diderot, Sorbonne Paris Cité, 75018 Paris, France

The selection of an antifungal regimen is based on multiple factors, including patients' characteristics, hospital setting, strain of Candida, and site of infection. The most appropriate antifungal regimen can be chosen from three main groups of antifungals: the azoles (mainly fluconazole), the polyenes (lipid formulation of amphotericin B), and the echinocandins (caspofungin, micafungin, anidulafungin). Although different antifungals can show comparable efficacy in treating candidemia and invasive candidiasis, their differences in terms of toxicity, drugdrug interactions, and selection of resistance remain significant and can impact the clinical outcome of fragile patient populations such as the critically ill. Thus, specific guidelines have been created in order to direct the clinicians in the challenging selection of the optimal antifungal therapy $[1,2]$. Among patients in the intensive care unit (ICU), crude mortality rates for invasive candidiasis can range from 25 to $60 \%$ [3, 4]. Although risk factors associated with ICU, e.g., use of broad-spectrum antibiotics, intravascular catheters, parenteral nutrition, and high Acute Physiology and Chronic Health Evaluation II (APACHE II) score, are frequently present in patients with candidemia [5], other hospital settings such as the internal medicine wards have shown increasing rates of Candida infections [6]. Candida colonization at one or more deep anatomical sites represents an important risk factor and frequently precedes invasive infections in non-neutropenic patients, and the rate of colonized patients in ICU increases as exposure to risk factors is prolonged [5]. In this regard, Pittet et al. have shown a relationship between the intensity of fungal colonization and the risk of invasive fungal infections (IFI) in ICU patients [7]. Several studies have reported the benefit of antifungal prophylactic therapy in colonized ICU patients to reduce IFI incidence, while many others failed to demonstrate any benefit in the same population [8-11]. The current study by Ferreira et al. [12] casts more significant doubts whether the extensive use of prophylactic antifungals in highly colonized patients can be considered safe and effective as a routine measure. In their retrospective observational study, the authors demonstrated that Candida colonization-based pre-emptive antifungal prescription of fluconazole and caspofungin generated a significant change in acquired colonization, especially with $C$. glabrata and $C$. parapsilosis, without any impact on incidence of candidemia and on Candida-related mortality. Furthermore it led to consumption of fluconazole and caspofungin of up to 240 and $170 \mathrm{DDD} / 1000$ ICU days, respectively, by far higher than those reported usually in ICU [13]. 
Fig. 1 Approach for antifungal therapy in non-neutropenic critically ill patients

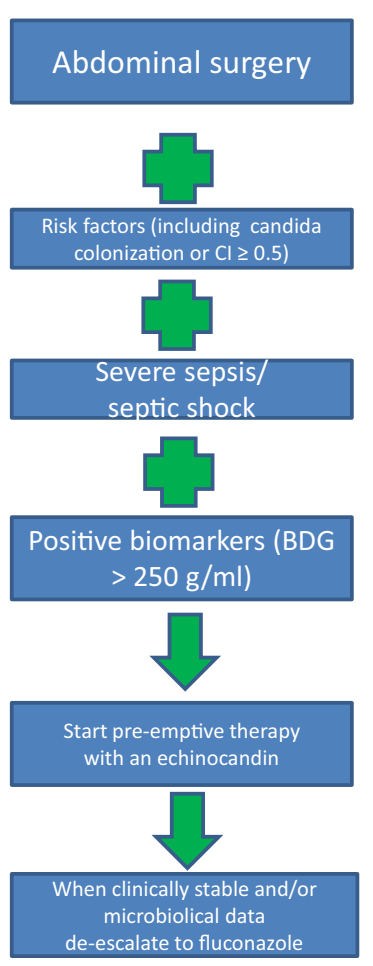

Even in previous studies, the colonization index's positive predictive value was less than $9 \%$; and in medical ICU patients, $39 \%$ developed a colonization index of more than 0.5 , while, in the same period, no invasive fungal infections were diagnosed [5]. The bedside practicality of the colonization index remains limited. In recent years, mainly as a result of the widespread use of fluconazole-based prophylaxis in ICU patients, the epidemiology of Candida spp. distribution has shifted from albicans (CA) to non-albicans (NAC) species, thus posing possible issues in terms of antifungal treatment [14].

A delay in the antifungal therapy has been linked to high mortality rates in different clinical contexts [15]. The lack of both adequate and timely antifungal treatment and source control have been linked to increased mortality in patients with septic shock due to Candida infection [16]. To minimize the negative impact of this infection, several management strategies have been proposed: prophylaxis, empirical therapy, pre-emptive therapy, and culture-based treatment. Both prophylaxis-based on universal antifungal treatment-and empirical therapy-based on the persistence of fever non-responsive to antibacterials and a combination of risk factors-may overexpose the patients to treatment, potentially increasing the rates of resistance to antifungals.

Although the benefits of antifungal prophylaxis are well established in neutropenic patients (e.g., hematological patients), the utility in non-neutropenic, critically ill patients is still controversial. Moreover, the empirical approach has limitations due to confounding features

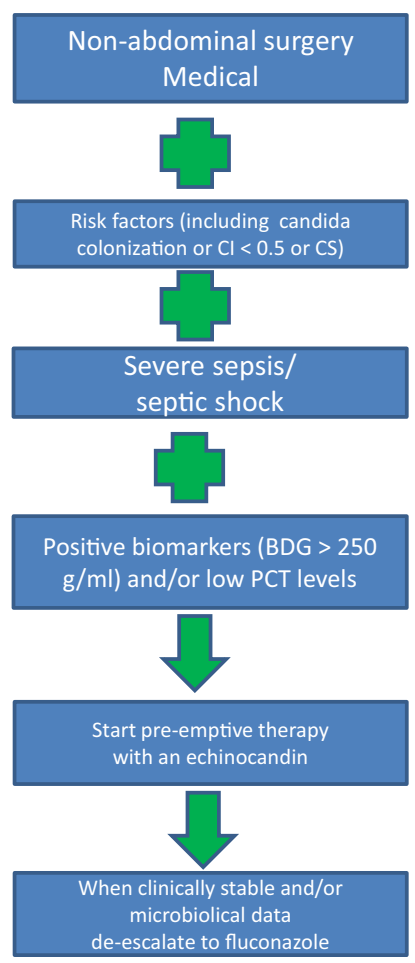

Anastomotic leakage Reopening of the digestive tract

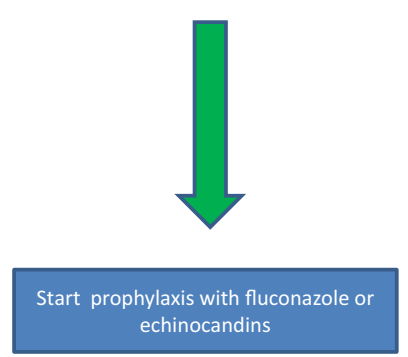

$\mathrm{Cl}$ : Colonization index, CS: Candida score; BDG: beta-D-glucan; PCT: procalcitonin. related to specific patient populations, the risk factors associated with candidemia being very common in ICU patients.

The use of colonization index to start a pre-emptive strategy is clearly inappropriate as a colonization index greater than 0.5 occurred in $26 \%$ of the patients and led to an overuse of antifungal without avoiding candidemia [12].

Predictive models of invasive candidiasis are useful to stratify critically ill patients at high risk but decisions regarding the use of antifungal treatment should not be exclusively based on these models. In recent years, nonculture diagnostic techniques based on serological biomarkers such as anti-mycelium antibodies (Candida albicans germ tube antibody test, CAGTA), 1,3- $\beta$-D-glucan (BDG), mannan antigen (Mn), and anti-mannan antibodies (AMn) have been developed, which may allow the differentiation of Candida spp. colonization and infection $[17,18]$. Thus, considering the use of this new marker of Candida infection and for practical purposes, it is proposed to substitute the concept of pre-emptive or empiric therapy by "early" antifungal treatment, in which both modalities would be present. The possible and "ideal" interdependence of microbiologic findings, clinical symptoms, and serologic and molecular biomarkers may help clinicians in the stratification of patients and to select more accurately those candidates for an "early" antifungal treatment. However, it is interesting to assess the reliability of these techniques, not only in cases of low-grade and high-grade colonization but also in 
candidemia (primary and catheter-related) and intra-abdominal candidiasis, considering all variables that may affect the results. This requires a practical operational classification, as it applies to immunosuppressed patients, that includes the concept of proven Candida infection microbiologically documented (candidemia and intra-abdominal candidiasis), and probable Candida infection based on the presence of high-risk patients (major abdominal surgery, pancreatitis), prolonged ICU stay, highgrade colonization, clinical condition of severe sepsis/ septic shock, and of positive results of specific biomarkers (preferably BDG) of candidal infection. Although blood cultures are characterized by a low sensitivity and a prolonged time to positivity (usually more than $72 \mathrm{~h}$ ) from the time of blood sample collection, they remain the essential investigation to assess candidemia. Targeted treatment is mandatory in case of yeast detection from blood culture [2].

We believe the combination of multiple organ failure, sepsis of unknown origin, BDG (if available) higher than $250 \mathrm{~g} / \mathrm{ml}$ or multiple colonization with Candida in mechanically ventilated patients for more than 7 days and receiving broad-spectrum antibacterial agents should represent a population with a particularly high risk of life- threatening invasive candidiasis and should receive some benefits from an antifungal treatment (Fig. 1). We suggest restricting antifungal prophylaxis to surgical patients presenting with anastomotic leakage after abdominal surgery or re-operation of the digestive tract during the same hospitalization in order to prevent the development of invasive candidiasis (Fig. 1).

Whereas targeted treatment is now clearly defined, the empirical/pre-emptive strategy is still controversial because the population that may benefit from this treatment is still poorly understood. Inclusion protocols combining patient type, clinical scores, and biomarkers should be used in randomized controlled trials in order to maximize our chance to demonstrate an effect on the outcome and to limit the overuse of antifungal therapy that promotes resistance.

Conflicts of interest MB serves on scientific advisory boards for Pfizer Inc, Merck, and Astellas Pharma Inc.; has received funding for travel or speaker honoraria from Pfizer Inc., Merck, Gilead Sciences, Teva Inc, and Astellas Pharma Inc. JFT serves on advisory board for Merck; has received funding for travel, institution grant, and speaker honoraria from Pfizer Inc., Astellas, Merck and Gilead Sciences. CL declares no conflict of interest.

\section{References}

1. Pappas PG, Kauffman CA, Andes D, Benjamin DK Jr, Calandra TF, Edwards JE Jr et al (2009) Clinical practice guidelines for the management of candidiasis: 2009 update by the Infectious Diseases Society of America. Clin Infect Dis 48:503-535

2. Cornely OA, Bassetti M, Calandra T, Garbino J, Kullberg BJ, Lortholary O et al (2012) ESCMID guideline for the diagnosis and management of Candida diseases 2012: non-neutropenic adult patients. Clin Microbiol Infect 18(Suppl 7):19-37

3. Zaoutis TE, Argon J, Chu J, Berlin JA, Walsh TJ, Feudtner C (2005) The epidemiology and attributable outcomes of candidemia in adults and children hospitalized in the United States: a propensity analysis. Clin Infect Dis 41:1232-1239

4. Leroy O, Gangneux JP, Montravers P Mira JP, Gouin F, Sollet JP et al (2009) Epidemiology, management, and risk factors for death of invasive Candida infections in critical care: a multicenter, prospective, observational study in France (2005-2006). Crit Care Med 37:1612-1618
5. Leon C, Ostrosky-Zeichner L, Schuster M (2014) What's new in the clinical and diagnostic management of invasive candidiasis in critically ill patients. Intensive Care Med 40:808-819

6. Bassetti M, Molinari MP, Mussap M, Viscoli C, Righi E (2013) Candidaemia in internal medicine departments: the burden of a rising problem. Clin Microbiol Infect 19:E281-E284

7. Pittet D, Monod M, Suter PM, Frenk E, Auckenthaler R (1994) Candida colonization and subsequent infections in critically ill surgical patients. Ann Surg 220:751-758

8. Garbino J, Lew DP, Romand JA, Hugonnet S, Auckenthaler R, Pittet D (2002) Prevention of severe Candida infections in nonneutropenic, high-risk, critically ill patients: a randomized, double-blind, placebo-controlled trial in patients treated by selective digestive decontamination. Intensive Care Med 28:1708-1717

9. Shorr AF, Chung K, Jackson WL, Waterman PE, Kollef MH (2005) Fluconazole prophylaxis in critically ill surgical patients: a meta-analysis. Crit Care Med 33:1928-1935
10. Albert M, Williamson D, Muscedere J, Lauzier F, Rotstein C, Kanji S, Jiang X, Hall M, Heyland D (2014) Candida in the respiratory tract secretions of critically ill patients and the impact of antifungal treatment: a randomized placebo controlled pilot trial (CANTREAT study). Intensive Care Med 40:1313-1322

11. Ostrosky-Zeichner L, Shoham S, Vazquez J et al (2014) MSG-01: a randomized, double-blind, placebocontrolled trial of caspofungin prophylaxis followed by preemptive therapy for invasive candidiasis in highrisk adults in the critical care setting. Clin Infect Dis 58:1219-1226

12. Ferreira D, Grenouillet F, Blasco G, Samain E, Hénon T, Dussaucy A, Millon L, Mercier M, Pili-Floury S (2015) Outcomes associated with routine systemic antifungal therapy in critically ill patients with Candida colonization. Intensive Care Med. doi:10.1007/s00134-015-3791-4 
13. Fournier P, Schwebel C, Maubon D, Vesin A, Lebeau B, Foroni L, Hamidfar-Roy R, Cornet M, Timsit JF, Pelloux H (2011) Antifungal use influences Candida species distribution and susceptibility in the intensive care unit. J Antimicrob Chemother 66(12):2880-2886

14. Bassetti M, Righi E, Costa A, Fasce R, Molinari MP, Rosso R et al (2006) Epidemiological trends in nosocomial candidemia in intensive care. BMC Infect Dis 6:21
15. Morrell M, Fraser VJ, Kollef MH (2005) Delaying the empiric treatment of Candida bloodstream infection until positive blood culture results are obtained: a potential risk factor for hospital mortality. Antimicrob Agents Chemother 49:3640-3645

16. Bassetti M, Righi E, Ansaldi F, Merelli M, Trucchi C, De Pascale G, DiazMartin A, Luzzati R, Rosin C, Lagunes L et al (2014) A multicenter study of septic shock due to candidemia: outcomes and predictors of mortality. Intensive Care Med 40:839-845
17. Eggimann P, Bille J, Marchetti O (2011) Diagnosis of invasive candidiasis in the ICU. Ann Intensive Care 1:37

18. Clancy CL, Nguyen MH (2014) Undiagnosed invasive candidiasis: incorporating non-culture diagnostics into rational prophylactic and preemptive antifungal strategies. Expert Rev Anti Infect Therapy 12:731-734 